\title{
In Silico Approach of Potential Phytochemical Inhibitor from Moringa oleifera, Cocos nucifera, Allium cepa, Psidium guajava, and Eucalyptus globulus for the treatment of COVID-19 by Molecular Docking
}

Ika Nur Fitriani ( $\square$ ikanurf@walisongo.ac.id )

Universitas Islam Negeri Walisongo Semarang

Wiji Utami

Universitas Islam Negeri Sulthan Thaha Saifuddin Jambi

Adi Tiara Zikri

Universitas Gadjah Mada

Pugoh Santoso

Kinki Daigaku Kyushu Tanki Daigaku

Research

Keywords: covid-19, in-silico, molecular docking, Moringa oleifera, Allium cepa, Cocos nucifera, Psidium guajava, Eucalyptus globulus

Posted Date: July 23rd, 2020

DOI: https://doi.org/10.21203/rs.3.rs-42747/v1

License: (c) (i) This work is licensed under a Creative Commons Attribution 4.0 International License.

Read Full License 


\section{Abstract \\ Background}

Coronavirus disease 2019 (COVID-19) is caused by infection with severe acute respiratory syndrome coronavirus 2. COVID-19 has devastating effects on people in all countries and getting worse. We aim to investigate an in-silico docking analysis of phytochemical compounds from medicinal plants that used to combat inhibition of the COVID-19 pathway. There are several phytochemicals in medicinal plants, however, the mechanism of bioactive compounds remains unclear. These results are obtained from in silico research provide further information to support the inhibition of several phytochemicals.

\section{Methods}

Molecular docking used to determine the best potential COVID-19 M pro inhibitor from several bioactive compounds in Moringa oleifera, Allium cepa, Cocos nucifera, Psidium guajava, and Eucalyptus globulus. Molecular docking was conducted and scored by comparison with standard drugs remdesivir. ADME properties of selected ligands were evaluated using the Lipinski Rule. The interaction mechanism of the most recommended compound predicted using the STITCH database.

\section{Results}

There was no recommended compound in Moringa oleifera as a potential inhibitor for COVID-19. Oleanolic acid in Allium cepa, a-tocotrienol in Cocos nucifera, asiatic acid in Psidium guajava and culinoside in Eucalyptus globulus were the most recommended compound in each medicinal plant. Oleanolic acid was reported to exhibit anti-COVID-19 activity with binding energy was $-9.20 \mathrm{kcal} / \mathrm{mol}$. This score was better than remdesivir as standard drug. Oleanolic acid interacted through the hydrogen bond with HIS41, THR25, CYS44, GLU166. Oleanolic acid binding with CASP-3, CASP-9, and XIAP signaling pathway.

\section{Conclusions}

Oleanolic acid in Allium cepa found as a potential inhibitor of COVID-19 M-pro that should be examined in future studies. These results suggest that oleanolic acid may be useful in COVID-19 treatment.

\section{Background}

COVID-19 first found in Wuhan China and become the pandemic in the world declared by theWorld Health Organization on 11 March $2020^{1}$. Coronavirus disease 2019 (COVID-19) is caused by infection with Severe Acute Respiratory Syndrome Corona Virus 2 (SARS-CoV-2). COVID-19 has devastating effects on people in all countries. Since 2019 n-CoV disrupts and damages the human immune system, causing 
varying degrees of damage to organs throughout the body ${ }^{2}$. By comparing coronavirus hosts infection trends in other vertebrates, SARS-CoV-2 was found to be identical SARS-CoV and MERS-CoV. SARS-CoV-2 may also be transmitted between humans. It can infect human cells by using human angiotensinconverting enzyme 2 (ACE2) as a receptor ${ }^{3}$.

The number of confirmed cases of COVID-19 has already exceeded 12.847.288 and 567.734 deaths worldwide on 12 July 2020 04:59 GMT, according to WHO, while estimates global mortality at 7\%. In condition, things are getting worse. Unfortunately, there is no specific antiviral treatment for the current pandemic. There is an urgent need to treat the infected patient and reduce mortality 4 .

Currently, there are no authorized vaccinations available for the prevention of SARS-CoV-2. Drug researchers and clinicians are working hard against COVID-19 to discover potential drugs. In the United States, remdesivir has been reported in improving the clinical condition for COVID-19 patients and has obtained emergency usage permission for the treatment of COVID-19. Therefore, potential chemotheraupetic agents are urgently needed to treat this disease ${ }^{5}$. However, there is much clinical research in China, but no drug has been approved for the treatment of COVID-19 ${ }^{6}$.

Medicinal plants have been used as pharmaceuticals because of the significant role in health care. Medicinal plants have antiviral activity, however its mechanism for the treatment of COVID-19 has not yet been explained. ${ }^{7}$. Bioactive compounds from phytochemicals become the source of therapeutic potential. Phytochemical has many spectra of chemical compounds with various function. Plants are believed to possess medicinal properties and have better compatibility than drugs in the human body 8 . Traditional medicine and the conventional method can turn into modern medicine after chemical and pharmaceutical screening ${ }^{7}$.

Studies of traditional medicinal plants have increased recently because the natural resources and variety of such plants enable them to complement modern pharmacological. As computer technology has developed, in silico approaches was widely used in efforts to elucidate the pharmacological basis of the functions of conventional medicinal plants. The application of virtual screening in drug discovery will enrich active compounds among the candidates and accurately indicate the mechanism of medicinal plants action, lowering costs, and increasing the effectiveness of the entire procedure. Several FDA approved drugs were developed by in silico approaches from natural herbs or animals ${ }^{9}$. Study of the molecular docking can be a simple gateway to the quest for successful natural drugs against these conditions ${ }^{10}$.

Guava (Psidium guajava) helps the body improve immunity. Flavonoids in guava fruit are responsible for antibacterial action by inhibiting bacterial replication in the body and also preventing bacterial adhesion to healthy body cells. Moringa olifera is a rich source of bioactive compounds that have many nutritional and pharmacological properties. Many of the works reveal that $M$. oleifera derived gist inhibits the initiation of the viral replication cycle. Moringa also used for anti-inflammatory ${ }^{11}$. Cocos nucifera has anti-oxidants, antidiabetic, bactericidal, and antiviral activities. Extracts of kopyor coconut were 
hypothesized and indicated for anti-itching, anti-bacterial, antioxidant, anti-viral, analgesic, and antiinflammatory drugs ${ }^{12}$. Onion (Allium cepa) contains quercetin, which has antiviral properties ${ }^{13}$. Eucalyptus globulus (eucalyptus) were evaluated against HSV-1 ${ }^{14}$.

COVID-19 is a highly contagious disease with high mortality, and there are no licensed drugs or vaccines yet. Computational methods used to design and manufacture drugs. Low time criteria for in silico approaches enable high throughput sampling of existing medicines to classify new drugs, and to forecast the adverse effects of novel drugs. However, clinical research is required. Computational chemistry has a significant role in investigating new medicines by molecular docking study in this work. The identification of specific targets now has grown through the use of computational tools to design new drugs ${ }^{15}$. We can find that the molecular docking experiment was carried out between several ligands and target for SARS-CoV-2 under specific method.

Computational methods can inform the design of experimental research. Recent work has demonstrated the usefulness of computational methods to find potential antiviral drugs of SARS-CoV-2. Protease in these viruses is crucial to hosting infection and is necessary for cell replication ${ }^{16}$. Molecular docking used to study the affinity between the ligands and the binding site. The purpose of this research is to conduct an in-silico docking analysis of phytochemical compounds from medicinal plants namely: Moringa oleifera, Allium cepa, Cocos nucifera, Psidium guajava, and Eucalyptus globulus that used to combat inhibition of COVID-19 pathway, with the hope will be a potential drug which has better activity.

\section{Method}

\section{Molecular Docking}

Binding mode of the phytoconstituents into the target of main protease (M-pro) was investigated using molecular docking study. The analysis was carried out in silico docking using AutoDock Tools 4 . Auto Dock 4.2 (Scripps Research Institute, La Jolla, CA, USA) was used to validate the compound's binding energy to the protein receptor. The 2D interactions diagram were retrieved from Discovery Studio 4.5 (Dassault Systemes BIOVIA, San Diego, CA, USA ${ }^{17}$. The bioactive compounds from five medicinal plants were obtained in the format .sdf from Pubchem compound database. Remdesivir as standard drug was selected from the drug bank in .sdf format from Pubchem. The comparative antiviral activity of the phytoconstituents of five herbal plants is analyzed by docking and binding energy ${ }^{18}$.

We performed docking simulation from several medicinal plants into 3CLpro-X77 as a protein receptor. The structure of 3CLpro-X77 was obtained from PDB using PBD ID in the database (PDB entry 6W63; www.rscb.org/pdb) ${ }^{19}$. Redocking of the co-crystal ligand X77 at SARS-CoV-2 Mpro active site was performed to optimize the molecular docking methods correctly ${ }^{20}$. Chimera 1.13 .1 used to prepare the ligands and the receptor protein ${ }^{21}$. All molecules of water were removed, and hydrogen atoms were added. The maximum numbers of generation and evaluation were set at 27000 and 250000 , respectively 22. The ligand was docked in the active site of SARS-CoV-2 M-pro. (PDB ID: 6W63). 
Autodock requires pre-calculated grid maps present in the docked ligand, one for each type of atom. The grid stores the potential energy resulting from the macromolecule interaction. The parameters for the Grid box were set using ADT. The Lamarckian Genetic can be validated by the ligand's best conformational with 50 docking runs for each ligand. The parameter was set as default. Ten ligand conformations in complex with the receptor were obtained after complete execution of AutoDock, which were ranked from binding energy 23 .

The lowest binding energy was calculated. Interaction of molecular such as hydrogen bonding, electrostatic, and hydrophobic interaction results was analyzed for structure-activity relationships ${ }^{24}$. Dock Score function was used to scoring all the dock ligands. Analyses from the best pose have been identified. In the current study, a docking score indicates the binding efficiency between the phytochemical constituents and the corresponding candidate targets for the treatment of COVID-19 inhibitor ${ }^{25}$.

\section{Lipinski Rule}

The restriction of ADME properties by Lipinski rule: a molecular weight less than 500 Daltons, hydrogen bond donors not more than 5 , hydrogen bond acceptors not more than 10 , and an octanol-water partition coefficient log P not greater than 5. Using DruLiTo software, selected ligands were tested for their Lipinski rule. DruLiTo aims to evaluate the ligand's pharmacokinetics and pharmacodynamic by checking the drug property. Important ADME properties (log P, H bond donor, H-bond acceptor, and molecular weight) are predicted. Based on Lipinski's rule in DruLiTo, the molecular properties and drug similarity of the phytochemical compounds were examined. ${ }^{26}$.

\section{STITCH Database}

An interface network between oleanolic acid-target was defined and established using the Search Tool for Interacting Chemicals (STITCH) database version 5.0 (http://stitch.embl.de/) ${ }^{27}$. The STITCH is a database of predicted interaction between protein and compound to explore the interaction between ligand and protein receptor ${ }^{28}$. Oleanolic acid was inserted in the STITCH database and set as default ${ }^{29}$. The species was set to Homo sapiens, and the molecular network interaction details could be collected.

\section{Results}

Several bioactive compounds from medicinal plants have been reported to exhibit antiviral bioactive. Several phytochemical inhibitors from Moringa oleifera, Allium cepa, Cocos nucifera, Psidium guajava, and Eucalyptus globulus have been investigated as potential COVID-19 inhibitors. The compounds pharmachopore compared to remdesivir as a standard drug from in-silico study. Previous studies have investigated the existence of 
abundant medicinal plants in nature. The binding energy obtained from docking of 6W63 protein with the native ligand was respectively $-9.24 \mathrm{kcal} / \mathrm{mol}$ (see Table1). The M-pro was used to build a model for the SARS-CoV-2 M-pro structure. The complex structure (6W63.pdb) determined from Xray crystallographic data, the inhibitor X77 was bonded to SARS-CoV-2 M-pro. AutoDock Tools has calculated the binding energy and binding mode of ligand X77 ${ }^{30}$.

Table 1. Docking results of compound Moringa oleifera with SARS-CoV-2 Mpro (6W63)

\begin{tabular}{lll}
\hline Compound name & Pubchem ID & Binding Energy (kcal/mol) \\
\hline native ligand & & -9.24 \\
sitogluside & 5742590 & -8.21 \\
\hline 4-hydroxybenzyl glucosinolate & 9601115 & -5.26 \\
\hline rhamnetin & 5281691 & -7.05 \\
\hline quercetin & 5280343 & -6.20 \\
\hline niazirin & 129556 & -6.59 \\
\hline niazimicin & 5471459 & -5.45 \\
\hline niaziminin & 10023860 & -6.09 \\
\hline kaempferol & 5280863 & -6.15 \\
\hline -sitosterol & 222284 & -7.65 \\
\hline remdesivir & 121304016 & -7.63 \\
\hline
\end{tabular}

The docking score between SARS-COV-2 M-pro and phytochemical compounds from Moringa oleifera: sitogluside, 4-hydroxybenzylglucosinolate, rhamnetin, quercetin, niazirin, niazimicin, kaempferol, and $\beta$-sitosterol was -8.21, -5.26, -7.05, -6.20, -6.59, -5.45, -6.09, and-7.65 respectively. The activity order was as follows: sitogluside $<\beta$-sitosterol < rhamnetin < niazirin < quercetin < niazimini < kaempferol < niazimicin < 4-hydroxybenzyl glucosinolate. The binding energy can be seen at Table 1. Sitogluside has the lowest binding energy. The lowest binding energy, the higher affinity. The docking score indicates the interaction affinity between enzyme and ligand by an optimized algorithm that acts as an inhibitory activity. In addition to phenolic hydroxyl, it may also affect their antiviral activities. In particular, various hydrophobic groups may make a significant contribution to binding with the hydrophobic cavity of the target proteins. The molecular docking results 
showed detailed information and the visual evidence of the ligand-inhibitor binding position. A similar binding position and binding mode may have the same inhibition mechanism.

Table 2. Residu interaction of Moringa oleifera with 6W63

\begin{tabular}{|c|c|c|c|}
\hline Compound name & Hydrogen bond & Electrostatic & Hydrophobic \\
\hline Native ligand & GLY143, CYS145, GLU166 & $\begin{array}{l}\text { MET 49, } \\
\text { HIS } 41\end{array}$ & $\begin{array}{l}\text { MET165, LEU27, } \\
\text { LEU141 }\end{array}$ \\
\hline Sitogluside & THR24, THR25, THR26 & - & $\begin{array}{l}\text { CYS145, HIS41, } \\
\text { MET49, MET165, } \\
\text { PRO168 }\end{array}$ \\
\hline $\begin{array}{l}\text { 4-Hydroxybenzyl } \\
\text { glucosinolate }\end{array}$ & $\begin{array}{l}\text { MET49, GLU166, ASN142, } \\
\text { THR190, GLN192, }\end{array}$ & - & ARG188 \\
\hline Rhamnetin & GLU166, GLN192, THR190 & - & $\begin{array}{l}\text { MET165, MET49, } \\
\text { PRO168 }\end{array}$ \\
\hline Quercetin & $\begin{array}{l}\text { GLU166, GLY143, ASN142, } \\
\text { SER144, ASP187 }\end{array}$ & - & HIS41, MET165 \\
\hline Niazirin & $\begin{array}{l}\text { GLU166, PHE140, SER144, } \\
\text { LEU141 }\end{array}$ & - & HIS41, MET165 \\
\hline Niazimicin & CYS44, THR25, HIS41, ARG188 & - & MET49 \\
\hline Niaziminin & $\begin{array}{l}\text { CYS44, MET49, GLU166, } \\
\text { ASN142 }\end{array}$ & - & $\begin{array}{l}\text { HIS41, MET165, } \\
\text { CYS145 }\end{array}$ \\
\hline Kaempferol & $\begin{array}{l}\text { GLU166, HIS143, GLY143, } \\
\text { CYS145, ARG188 }\end{array}$ & - & MET165 \\
\hline$\beta$-sitosterol & - & - & $\begin{array}{l}\text { LEU167, PRO168, } \\
\text { MET165, HIS41, } \\
\text { CYS145 }\end{array}$ \\
\hline Remdesivir & $\begin{array}{l}\text { GLU166, ASN142, CYS145, } \\
\text { ASP187, GLN189 }\end{array}$ & - & MET49, HIS41 \\
\hline
\end{tabular}

The docking analysis (Table 2) showed that remdesivir forms H-bonds with the Glu166, ASN142, CYS145, ASP187, GLN189 (figure 2A). All of the H-bonds in the Covid-19 Mpro active site interacted with amino acid. The affinity of drug candidates depends upon the type and number of bonding occurring in protein active sites. In Table 2 remdesivir forms hydrogen bonds and hydrophobic bonds with 6W63 protein. Chemical bonds in rhamnetin, quercetin, niazirin, niaziminin, and kaempferol similar to native ligand. The highest affinity of all the compounds was sitogluside bond, but there is no similar H-bond with the native ligand. The second highest compound was $\beta$-sitosterol, but there is no 
similar residue also. Rhamnetin has a similar H-bond, but remdesivir as standard drug has a higher binding energy score. There was no recommended compound in Moringa oleifera as potential inhibitor for COVID-19.

Table 3.Docking results of compound Allium cepa with SARS-COV-2 Mpro (6W63)

\begin{tabular}{lll}
\hline Compound name & Pubchem ID & Binding Energy (kcal/mol) \\
\hline cyanidin 4'-glucoside & 73981555 & -5.78 \\
cyanidin 3-O-glucoside & 441667 & -7.03 \\
\hline oleanolic acid & 10494 & -9.20 \\
\hline (-)-jasmonic acid & 5281166 & -5.43 \\
\hline kaempferol 4'-glucoside & 5491693 & -6.27 \\
\hline methyl ferulate & 5357283 & -5.19 \\
\hline peonidin 3-arabinoside & 91810651 & -7.08 \\
\hline progesterone & 5994 & -8.38 \\
\hline quercitrin & 5280459 & -7.19 \\
\hline remdesivir & 121304016 & -7.63 \\
\hline
\end{tabular}

The docking score between SARS-CoV-2 M-pro and phytochemical compounds from Allium cepa: 9 cyanidin 4'-glucoside, cyanidin 3-O-glucoside, oleanolic acid, (-)-jasmonic acid, kaempferol 4'-glucoside, methyl ferulate, peonidin 3-arabinoside, progesteron, and quercitrin was $-5.78,-7.03,-9.20,-5.43,-6.27,-5.19,-7.08,-8.38$ and-7.19 respectively. The activity order was as follows: oleanolic acid < progesterone < quercitrin < peonidin 3arabinoside < cyanidin 3-O-glucoside < kaempferol 4'-glucoside < cyanidin 4'-glucoside < Methyl ferulate. The binding energy can be seen at Table 3 .

Table 3. Residu interaction of Allium cepa with 6W63 


\begin{tabular}{|c|c|c|}
\hline $\begin{array}{l}\text { Compound } \\
\text { name }\end{array}$ & $\begin{array}{l}\text { Hydrogen } \\
\text { Bond }\end{array}$ & Hydrophobic \\
\hline $\begin{array}{l}\text { Native } \\
\text { ligand }\end{array}$ & $\begin{array}{ll}\text { GLY143, } & \text { MET } 49, \text { HIS } 41 \\
\text { CYS145, } & \\
\text { GLU166 } & \end{array}$ & $\begin{array}{l}\text { MET165, } \\
\text { LEU27, } \\
\text { LEU141 }\end{array}$ \\
\hline $\begin{array}{l}\text { cyanidin } 4^{\prime}- \\
\text { glucoside }\end{array}$ & $\begin{array}{l}\text { GLY143, THR26, ASN142, THR25, } \\
\text { GLN192, THR190, GLU166 }\end{array}$ & - \\
\hline $\begin{array}{l}\text { cyanidin } 3-\mathrm{O} \\
\text { glucoside }\end{array}$ & $\begin{array}{l}\text { GLU166, SER144, HIS163, GLY143, } \\
\text { THR26, HIS41 }\end{array}$ & CYS145 \\
\hline oleanolic aci & HIS41, THR25, CYS44, GLU166 & $\begin{array}{l}\text { CYS145, MET49, } \\
\text { MET165, LEU167, } \\
\text { PRO168 }\end{array}$ \\
\hline $\begin{array}{l}(-) \text {-jasmonic } \\
\text { cid }\end{array}$ & ARG188 & $\begin{array}{l}\text { MET49, CYS44, } \\
\text { PRO52 }\end{array}$ \\
\hline $\begin{array}{l}\text { kaempferol } \\
\text { 4'-glucoside }^{\text {'-glid }}\end{array}$ & $\begin{array}{l}\text { GLU166, PHE140, ASN142, THR25, } \\
\text { HIS41 }\end{array}$ & HIS41, THR25 \\
\hline $\begin{array}{l}\text { methyl } \\
\text { ferulate }\end{array}$ & THR190, GLN192 & $\begin{array}{l}\text { LEU167, PRO168, } \\
\text { MET165 }\end{array}$ \\
\hline $\begin{array}{l}\text { peonidin 3-a } \\
\text { abinoside }\end{array}$ & THR26, GLY143, GLU166 & MET165, LEU141 \\
\hline progesteron & GLY143, GLN192, THR190 & MET165 \\
\hline quercitrin & $\begin{array}{l}\text { GLU166, GLY143, THR26, PHE140, } \\
\text { GLN189 }\end{array}$ & MET49, MET145 \\
\hline remdesivir & $\begin{array}{l}\text { GLU166, ASN142, CYS145, ASP187, } \\
\text { GLN189 }\end{array}$ & MET49, HIS41 \\
\hline
\end{tabular}

Chemical bonds in ligand cyanidin 4'-glucoside, cyanidin 3-O-glucoside, oleanolic acid, kaempferol 4'-glucoside, peonidin 3-arabinoside, progesteron and quercitrin similar to native ligand. The highest affinity of all the compounds was oleanolic acid. The docking 
analysis showed that several compounds were inhibited ranked by binding energy showed affinity $(\Delta \mathrm{G})$. The most recommended compound in Allium cepa was oleanolic acid.

Table 5. Docking result of compound Cocos nucifera with SARS-CoV-2 Mpro (6W63)

\begin{tabular}{lll}
\hline Compound name & Pubchem ID & Binding Energy (kcal/mol) \\
\hline 9-ribosyl-trans-zeatin & 6440982 & -7.16 \\
trans-zeatin & 449093 & -4.64 \\
\hline stigmasterol & 5280794 & -9.40 \\
\hline fucosterol & 5281328 & -9.14 \\
\hline$\beta$-tocotrienol & 5282348 & -8.14 \\
$\alpha$-tocotrienol & 5282347 & -8.59 \\
\hline$\beta$-tocopherol & 6857447 & -7.97 \\
$\alpha$-tocopherol & 14985 & -7.84 \\
\hline remdesivir & 121304016 & -7.63 \\
\hline
\end{tabular}

The docking score between SARS-CoV-2 M-pro and phytochemical compounds from Cocos nucifera: 9-ribosyl-trans-zeatin, trans-zeatin, stigmasterol, fucosterol, $\beta$-Tocotrienol, $\alpha-$ tocotrienol, $\beta$-tocopherol, and $\alpha$-tocopherol was -7.16, -4.64, -9.40, -9.14, -8.14, -8.59, -7.97, -7.84 and-7.63 respectively. The activity order was as follows: stigmasterol $<$ fucosterol $<$ $\alpha$-tocotrienol $<\beta$-tocotrienol $<\beta$-tocopherol $<\alpha$-tocopherol $<9$-ribosyl-trans-zeatin $<$ trans-zeatin. The binding energy can be seen at Table 5 .

Table 6. Residu interaction of Cocos nucifera with 6W63 


\begin{tabular}{|c|c|c|c|}
\hline $\begin{array}{l}\text { Compound } \\
\text { name }\end{array}$ & Hydrogen bond & Electrostatic & Hydrophobic \\
\hline $\begin{array}{l}\text { native } \\
\text { ligand }\end{array}$ & GLY143, CYS145, GLU166 & $\begin{array}{l}\text { MET 49, } \\
\text { HIS } 41\end{array}$ & MET165, LEU27, LEU141 \\
\hline $\begin{array}{l}\text { 9-ribosyl- } \\
\text { trans-zeatin }\end{array}$ & $\begin{array}{l}\text { GLU166, THR190, } \\
\text { ARG188, MET49, HIS41 }\end{array}$ & - & CYS44 \\
\hline trans-zeatin & TYR54, THR190 & - & MET165, MET49, THR190 \\
\hline stigmastero & - & - & $\begin{array}{l}\text { MET165, MET49, CYS44, } \\
\text { CYS145, HIS41 }\end{array}$ \\
\hline fucosterol & PHE149, ASN142 & - & $\begin{array}{l}\text { MET49, MET165, LEU167, } \\
\text { PRO168, HIS41, CYS145 }\end{array}$ \\
\hline $\begin{array}{l}\beta \text {-tocotrien } \\
\text { ol }\end{array}$ & GLY143, LEU141 & - & $\begin{array}{l}\text { HIS41, MET165, HIS163, } \\
\text { HIS172, CYS145 }\end{array}$ \\
\hline $\begin{array}{l}\alpha- \\
\text { tocotrienol }\end{array}$ & GLU166, PHE140 & - & $\begin{array}{l}\text { PRO168, LEU167, MET165, } \\
\text { HIS41, MET49, CYS145, HIS163 }\end{array}$ \\
\hline $\begin{array}{l}\beta- \\
\text { tocopherol }\end{array}$ & GLN192, THR190 & - & $\begin{array}{l}\text { PRO168, MET165, CYS145, } \\
\text { HIS163, HIS41, MET49 }\end{array}$ \\
\hline $\begin{array}{l}\alpha \text {-tocopher } \\
\text { ol }\end{array}$ & GLU166 & - & $\begin{array}{l}\text { MET49, HIS163, MET165, } \\
\text { HIS41 }\end{array}$ \\
\hline remdesivir & $\begin{array}{l}\text { GLU166, ASN142, } \\
\text { CYS145, ASP187, GLN189 }\end{array}$ & - & MET49, HIS41 \\
\hline
\end{tabular}

The affinity of drug candidate depends upon the type and number of bonding occurring in protein active site. Amino acid residue interaction in ligand 9-ribosyl-transzeatin, $\alpha$-tocotrienol, and $\alpha$-tocopherol, similar to native ligand. The highest affinity of all the compounds was stigmasterol bond or higher than other compounds, but there is no hydrogen bond in amino acid residue. The docking analysis showed that several compounds were inhibited ranked by binding energy showed affinity $(\Delta \mathrm{G})$. The most recommended compound in Cocos nucifera was $\alpha$-tocotrienol with binding energy score $-8.59 \mathrm{kcal} / \mathrm{mol}$.

Table 7. Docking result of compound Psidium guajava with SARS-CoV-2 Mpro (6W63) 


\begin{tabular}{lll}
\hline Compound name & Pubchem ID & Binding Energy (kcal/mol) \\
\hline arjunolic acid & 73641 & -8.09 \\
asiatic acid & 119034 & -8.31 \\
\hline avicularin & 54900064 & -5.90 \\
\hline 3-O-beta-D-glucopyranosyl sitosterol & 79699351 & -8.14 \\
\hline guaijaverin & 5481224 & -6.49 \\
\hline guavanoic acid & 01211343 & -8.30 \\
\hline morin & 5281670 & -6.29 \\
\hline leucocyanidin & 44083 & -6.54 \\
\hline
\end{tabular}

The docking score between SARS-COV-2 main protease and phytochemical compounds from Psidium guajava: arjunolic acid, asiatic acid, avicularin, 3-O-beta-Dglucopyranosyl sitosterol, guaijaverin, guavanoic acid, morin, and leucocyanidin was -8.09, $-8.31,-5.90,-8.14,-6.49,-6.30,-8.30,-6.29$ and -6.54 respectively. The activity order was as follows: Asiatic acid < guavanoic acid < 3-O-beta-D-Glucopyranosyl sitosterol < arjunolic acid < leucocyanidin guaijaverin $<$ morin $<$ avicularin. The binding energy can be seen at Table 7.

Table 8. Residu interaction of Psidium guajava with 6W63 


\begin{tabular}{|c|c|c|c|}
\hline Compound name & Hydrogen bond & Electrostatic & Hydrophobic \\
\hline native ligand & GLY143, CYS145, GLU166 & $\begin{array}{l}\text { MET 49, } \\
\text { HIS 41 }\end{array}$ & $\begin{array}{l}\text { MET165, LEU27, } \\
\text { LEU141 }\end{array}$ \\
\hline arjunolic acid & $\begin{array}{l}\text { GLN192, THR190, GLY143, } \\
\text { ASN142 }\end{array}$ & - & $\begin{array}{l}\text { PRO168, LEU167, } \\
\text { MET165, MET49, } \\
\text { CYS145, HIS41 }\end{array}$ \\
\hline asiatic acid & GLU166, THR45, HIS41 & - & $\begin{array}{l}\text { PRO168, MET49, } \\
\text { MET165, CYS145 }\end{array}$ \\
\hline avicularin & $\begin{array}{l}\text { PHE140, GLU166, ASN142, } \\
\text { THR26 }\end{array}$ & - & LEU141 \\
\hline $\begin{array}{l}\text { 3-O-beta-D-glucopy } \\
\text { ranosyl sitosterol }\end{array}$ & GLU166, PRO168 & - & $\begin{array}{l}\text { CYS145, CYS44, } \\
\text { HIS41, MET165 }\end{array}$ \\
\hline guaijaverin & $\begin{array}{l}\text { GLU166, GLN189, PHE140, } \\
\text { GLY143, THR26 }\end{array}$ & - & HIS41 \\
\hline guavanoic acid & THR190, GLN192, MET49 & - & $\begin{array}{l}\text { LEU27, CYS145, } \\
\text { MET165, HIS41, } \\
\text { LEU167 }\end{array}$ \\
\hline morin & $\begin{array}{l}\text { CYS145, GLY143, HIS163, } \\
\text { GLU166, ARG188 }\end{array}$ & - & MET165 \\
\hline leucocyanidin & $\begin{array}{l}\text { GLY143, ASN142, SER144, } \\
\text { HIS163, GLU166, ARG188 }\end{array}$ & - & MET165 \\
\hline remdesivir & $\begin{array}{l}\text { GLU166, ASN142, CYS145, } \\
\text { ASP187, GLN189 }\end{array}$ & - & MET49, HIS41 \\
\hline
\end{tabular}

Residue interaction in ligand arjunolic acid, asiatic acid, avicularin, 3-O-beta-Dglucopyranosyl sitosterol, guaijaverin, morin, and leucocyanidin, similar to standard drug/native ligand. The highest affinity of all the compounds was Asiatic acid bond or higher than other compounds. The most recommended compound in Psidium guajava was asiatic acid with binding energy score $-8.31 \mathrm{kcal} / \mathrm{mol}$.

Table 9. Docking results of compound Eucalyptus globulus with SARS-CoV-2 Mpro (6W63)

\begin{tabular}{lll}
\hline Compound name & Pubchem ID & Binding Energy (kcal/mol) \\
\hline quercetin 3-o-l-rhamnoside & 5280459 & -6.69 \\
8-C-glucosylquercetin 2"-O-rhamnoside & 44257960 & -6.14 \\
cuniloside & 10052127 & -8.58 \\
\hline cypellocarpin a & 10696653 & -6.60 \\
euglobal-ia1 & 442511 & -7.65 \\
\hline gibberellin a1 & 5280379 & -7.74 \\
globulusin b & 24829134 & -7.00 \\
myricetin & 5281672 & -6.36 \\
\hline eucalyptol & 46506161 & -5.38 \\
\hline
\end{tabular}


The docking score between SARS-CoV-2 M-pro and phytochemical compounds from Eucalyptus globulus: quercetin 3-O-L-rhamnoside, 8-C-glucosylquercetin 2"-O-rhamnoside, cuniloside, cypellocarpin A, euglobal-Ia1, gibberellin A1, globulusin B, and myricetin was $-6.69,-6.14,-8.58,-6.60,-7.65,-7.74,-7.00,-6.60$ and -6.36 respectively. The activity order was as follows: cuniloside < gibberellin A1< euglobal-la1 < globulusin B < quercetin 3-O-Lrhamnoside < cypellocarpin A $<$ myricetin < 8-C-glucosylquercetin 2"-O-rhamnoside. Quercetin is a flavonol and is found in many fruits and vegetables. The antioxidant properties may be responsible for the pharmacological activity of quercetin ${ }^{31}$. The binding energy can be seen at Table 9 .

Table 10. Residu interaction of Eucalyptus globulus with 6W63 


\begin{tabular}{|c|c|c|c|}
\hline Compound name & Hydrogen bond & Electrostatic & Hydrophobic \\
\hline native ligand & $\begin{array}{l}\text { GLY143, CYS145, } \\
\text { GLU166 }\end{array}$ & $\begin{array}{l}\text { MET 49, } \\
\text { HIS } 41\end{array}$ & MET165, LEU27, LEU141 \\
\hline $\begin{array}{l}\text { quercetin 3-o-l- } \\
\text { rhamnoside }\end{array}$ & $\begin{array}{l}\text { HIS41, CYS145, } \\
\text { GLY143, GLU166 }\end{array}$ & - & CYS44, MET49 \\
\hline $\begin{array}{l}\text { 8-C-glucosylquerceti } \\
\text { n 2"-O-rhamnoside }\end{array}$ & $\begin{array}{l}\text { THR190, GLU166, } \\
\text { ASN142, HIS164, } \\
\text { SER144 }\end{array}$ & - & MET165, HIS163 \\
\hline cuniloside & $\begin{array}{l}\text { GLU166, ASN142, } \\
\text { GLN192, THR190 }\end{array}$ & - & MET165 \\
\hline cypellocarpin a & $\begin{array}{l}\text { GLU166, ASN142, } \\
\text { HIS41, THR25 }\end{array}$ & - & MET49, CYS145 \\
\hline euglobal-ia1 & GLU166 & - & $\begin{array}{l}\text { CYS145, HIS41, MET49, } \\
\text { CYS44, PRO52, TYR54 }\end{array}$ \\
\hline gibberellin a1 & $\begin{array}{l}\text { ARG188, GLU166, } \\
\text { GLN189 }\end{array}$ & - & MET165, HIS41, PRO168 \\
\hline globulusin b & $\begin{array}{l}\text { GLY143, LEU141, } \\
\text { SER144, ASN142, } \\
\text { ARG188 }\end{array}$ & - & MET165 \\
\hline Myricetin & $\begin{array}{l}\text { GLU166, ASN142, } \\
\text { ARG188, GLN192, } \\
\text { THR190 }\end{array}$ & - & PRO168, MET165 \\
\hline Eucalyptol & GLN189 & - & $\begin{array}{l}\text { MET49, CYS44, HIS41, } \\
\text { MET165, ARG188, } \\
\text { PRO52, TYR54 }\end{array}$ \\
\hline Remdesivir & $\begin{array}{l}\text { GLU166, ASN142, } \\
\text { CYS145, ASP187, } \\
\text { GLN189 }\end{array}$ & - & MET49, HIS41 \\
\hline
\end{tabular}

Residue interaction in ligand quercetin 3-O-L-rhamnoside, 8-C-glucosylquercetin 2"-Orhamnoside, cypellocarpin A, euglobal-Ia1, gibberellin A1, globulusin B, and myricetin, similar to native ligand. The highest affinity of all the compounds was cuniloside bond, or higher than other compounds. The most recommended compound in Eucalyptus globulus was cuniloside with binding energy score $-8.58 \mathrm{kcal} / \mathrm{mol}$. Residue interaction of most recommended compounds in each plant can be seen in Figure 1.

\section{Discussion}

In this work, the most effective drugs were chosen to suggest possible treatment of COVID-19 based on molecular docking compared with the ligand X77 and standard drug. The docking score showed the ligand's binding energy from several phytochemical with 
target protein SARS-CoV-2 Mpro using Auto Dock Tools. Docking studies are used to investigate plant-receptor interaction. Complex stability retrieved from docking score and visualization of amino acid residue. The phytochemical compounds were analyzed using AutoDock Tools.

According to the $\mathrm{WHO}$, due to its safety and medicinal capacity, most countries rely on herbal products ${ }^{32}$. The secondary metabolite compounds from plants in tropical regions can be developed as medicines. Several bioactive compounds reported have antiviral activity. In this research, we investigated several phytochemicals as a potential candidate for the COVID-19 M-pro inhibitor.

The finding from this research will give opportunities to develop the right drug for COVID-19 M-pro from protein with PDB ID 6W63. Ligands and several drug candidate compounds from the best docking result in each plant were evaluated further on the Lipinski rule. Novel drug design was developed using chemo-informatics based on the phytochemical lead structure. Lipinski's rule of five was determined using DruLiTo. The physico-chemical parameter calculated, such as molecular weight, partition coefficient, hydrogen bonds donor, and acceptor ${ }^{33}$. Drug candidate selected from ligands which did not have more than two violations. Lipinski rule of five accepted all tested compounds in this study. Lipinski Rule can be seen in table 11.

Table 11. Lipinski rule of selected ligands

\begin{tabular}{|c|c|c|c|c|c|c|c|}
\hline No & Compound & $\begin{array}{l}\text { Molecular } \\
\text { weight }<500\end{array}$ & $\begin{array}{l}\log P \\
<5\end{array}$ & $\begin{array}{l}\text { H bond } \\
\text { donor (5) }\end{array}$ & & $<10$ & Violation \\
\hline 1 & $\begin{array}{l}\text { Oleanolic } \\
\text { acid }\end{array}$ & 456.36 & 9.052 & 2 & 3 & 1 & \\
\hline 2 & $\alpha$-tocotrienol & 424.33 & 7.914 & 1 & 2 & 1 & \\
\hline 3 & Asiatic acid & 488.35 & 7.439 & 4 & 5 & 1 & \\
\hline 4 & Cuniloside & 512.26 & 0.913 & 5 & 10 & 2 & \\
\hline
\end{tabular}


Phytochemical screened can compete with the substrate based on scoring function, residue interaction, and drug-like properties. The parameter can inhibit macro domain function, eventually losing SARS-COV-2 virus virulence ${ }^{34}$. All the compounds accepted the Lipinski rule. The highest binding energy from 4 compounds was oleanolic acid. Network interaction in oleanolic acid analyzed further using STITCH Database. The oleanolic acid compound from Allium cepa had significant interaction with the target protein could be further analyzed with molecular dynamics and in vitro methods.

Viruses need host protein to replicate themselves before hijack the host cell. Therefore, viral replication can be blocked using the host protein inhibitor. The host protein inhibitor will be antiviral drug ${ }^{35}$. Several drugs are predicted to be used in the treatment of COVID-19 but still debated ${ }^{36}$. However, most in vitro and in vivo experiments did not specify the molecular target of these secondary metabolites, and the pathways for the functions of plant compounds remain uncertain. The pharmacophore model is valid for simultaneous screening to predict and simulate the binding condition of compounds and targets ${ }^{37}$.

Oleanolic acid interface network focused on an overview of the STITCH index. The first shell had ten nodes, UGT2B11, UGT2B10, NFE2L2, NQO1, PPARA, PTGIR, NAMPYT, CASP3, TOP2A, and TOP1; the second shell had ten nodes, topotecan, camptothecin, XIAP, BIRC2, CASP9, rosiglitazone, NCOR1, MAFG, KEAP1, and dicumarol. The top two antiCOVID19 related KEGG pathways with oleanolic acid-target genes were the CASP3, CASP9, and XIAP signaling pathways. Network interaction for this predictive was activation, inhibition, and catalysis.

Oleanolic acid has a higher binding potential to SARS-CoV-2 3CL M-pro than remdesivir with COVID-19. These may be paired with ACE2 binding to CASP-3 signaling pathway to affect apoptosis, which is deserving of more study and aims to provide analytical guidance. Recent molecular docking results revealed a high affinity of oleanolic acid with the target proteins. The chemical compounds involved various biological processes and pathways for the treatment of COVID-19 by combining with essential target 
proteins CASP-3, CASP-9, and XIAP, which supported the clinical treatment of COVID-19. Interaction network can be seen in Figure 2.

Oleanolic acid has antiviral effects. Based on the STITCH database, XIAP (X-linked apoptosis inhibitor) is multifunctional protein that regulates caspase and apoptosis, and modulates inflammatory signal and immunity, cell proliferation, cell invasion, and metastasis. XIAP act as a direct inhibitor of caspase. Caspase 3, apoptosis-related cycteine peptidase, active in triggering of caspases responsible for the execution of apoptosis. Caspase 9, apoptosis-related peptidase cysteine. Oleanolic acid has KEGG pathways viral myocarditis with pathway ID 05416. Based on the previous study, oleanolic acid mediated apoptosis requiring the release of mitochondria cytochrome $\mathrm{C}$ into the cytosol and triggered the activation of caspase- 9 and caspase-3 accompanied by polymerase (ADPribose) polymerase (PARP cleavage) ${ }^{38}$.

Oleanolic acid was the most recommended compound in medicinal plants, which can be a potential inhibitor for COVID-19. This research rationalize the limited data on drug efficacy for COVID-19 treatment and give information to select drug candidate for in-vitro trials and in-vivo test. The predicted drug binding and ranking will also be useful in interpreting the results of clinical experiments against COVID-19.

\section{Conclusion}

In silico approach of herbal medicine for COVID-19 reported as well. Allium cepa is potent herbal medicine for the development of new therapeutics for COVID-19. Oleanolic acid was the most recommended compound in medicinal plants, which can be a potential inhibitor for COVID-19. Further research is required to examine the potential uses of medicinal plants. These results suggest that antiviral and anti-inflammatory activities from oleanolic acid may be useful in COVID-19 treatment.

\section{Abbreviations}

ACE2

angiotensin-converting enzyme 2

BIRC2

baculoviral IAP repeat containing 2

CASP9

caspase 9

CASP3 
caspase 3

COVID-19

Coronavirus disease 2019

KEAP1

kelch-like $\mathrm{ECH}$-associated protein 1

KEGG

Kyoto Encyclopedia of Genes and Genome

MAFG

v-maf musculoaponeurotic fibrosarcoma oncogene homolog G (avian)

M-pro

main protease

NAMPT

nicotinamide phosphoribosyltransferase

NCOR1

nuclear receptor corepressor 1

NFE2L2

nuclear factor (erythroid-derived 2)-like 2

NQ01

NAD $(P) H$ degydrogenase, quinone 1

PARP

Poly (ADP-ribose) polymerase

PPARA

peroxisome proliferator-activated receptor alpha

PTGIR

prostaglandin 12 receptor

SARS-CoV-2

Severe Acute Respiratory Syndrome Coronavirus 2

STITCH

Search Tool for Interacting Chemicals

TOP1

topoisomerase (DNA) I

TOP2A

topoisomerase (DNA) II alpha

UGT2B11

UDP glucuronosyltransferase 2 family, polypeptide B11

UGT2B10

UDP glucuronosyltransferase 2 family, polypeptide B11

XIAP

X-linked inhibitor of apoptosis 


\section{Declarations}

- Ethics approval and consent to participate

Not applicable

- Consent for publication

Not applicable

- Availability of data and materials

Not applicable

- Competing interests

The authors declare that they have no competing interests

- Funding

No funding

- Author's contribution

F-IN, UW, Z-AT, SP wrote the manuscript. All authors read and approved the final manuscript.

- Acknowledgements

Not applicable

\section{References}

1. Chen YW, Yiu CPB, Wong KY. Prediction of the SARS-CoV-2 (2019-nCoV) 3C-like protease (3CL pro) structure: virtual screening reveals velpatasvir, ledipasvir, and other drug repurposing candidates. F1000Research. 2020;9:129. doi:10.12688/f1000research.22457.1

2. Ruan X, Du P, Zhao K, et al. Mechanism of Dayuanyin in the treatment of coronavirus disease 2019 based on network pharmacology and molecular docking. Chin Med. 2020;15(1):1-17. doi:10.1186/s13020-020-00346-6

3. Tong T, Wu YQ, Ni WJ, Shen AZ, Liu S. The potential insights of Traditional Chinese Medicine on treatment of COVID-19. Chinese Med (United Kingdom). 2020;15(1):1-6. doi:10.1186/s13020-02000326-w 
4. Lee K, Mun S, Pyun H, Kim M, Hwang J. Effects of Macelignan Isolated from Myristica fragrans ( Nutmeg ) on Expression of Matrix Metalloproteinase-1 and Type I Procollagen in UVB-Irradiated Human Skin Fibroblasts. 2012;35(October):1669-1675.

5. da Silva JKR, Figueiredo PLB, Byler KG, Setzer WN. Essential oils as antiviral agents. Potential of essential oils to treat sars-cov-2 infection: An in-silico investigation. Int J Mol Sci. 2020;21(10). doi:10.3390/ijms21103426

6. Shi Y, Zhang X, Mu K, et al. D3Targets-2019-nCoV: a webserver for predicting drug targets and for multi-target and multi-site based virtual screening against COVID-19. Acta Pharm Sin B. 2020. doi:10.1016/j.apsb.2020.04.006

7. Savithramma N, Rao ML, Suhrulatha D. Screening of Medicinal Plants for Secondary Metabolites. 2011;8(3):579-584.

8. Nagamani C, Devi K, Sridharbabu G, Rajashekar V, Enaganti S. Molecular docking studies of synthetic and natural compounds against cFLIP protein in cancer. J App/ Pharm Sci. 2017;7(3):109112. doi:10.7324/JAPS.2017.70317

9. Yi F, Li L, Xu L jia, et al. In silico approach in reveal traditional medicine plants pharmacological material basis. Chinese Med (United Kingdom). 2018;13(1):1-20. doi:10.1186/s13020-018-0190-0

10. Mohapatra S, Prasad A, Haque F, Ray S, De B, Ray SS. In silico investigation of black tea components on a-amylase, a-glucosidase and lipase. J Appl Pharm Sci. 2015;5(12):42-47. doi:10.7324/JAPS.2015.501207

11. Bhardwaj R, Pareek S, Sagar NA, Vyas N. Bioactive Compounds in Underutilized Fruits and Nuts.; 2019. doi:10.1007/978-3-030-06120-3

12. Hariyadi DM, Rosita N, Purwokerto UM. INTERNATIONAL JOURNAL OF RESEARCH IN Characterization and antibacterial activity of cocos Nucifera L . Meat. 2020;11(1):611-616.

13. Das M, Banerji A, Cheemalapati VN, et al. Review Paper ANTIVIRAL ACTIVITY OF INDIAN MEDICINAL PLANTS : PRVENTIVE MEASURES FOR COVID-19. 2020;09(5):7307-7319.

14. Mohamed Nadjib B. Effective Antiviral Activity of Essential Oils and their Characteristic Terpenes against Coronaviruses: An Update. J Phar-macol Clin Toxicol. 2020;8(1):1138.

15. Sekhar T. Virtual Screening based prediction of potential drugs for COVID-19. 2020;(March). doi:10.20944/preprints202002.0418.v2

16. Mevada VA, Dudhagara PR, Patel R. Drug Repurposing of Approved Drugs Elbasvir, Ledipasvir , Paritaprevir, Velpatasvir , Antrafenine and Ergotamine for Combating COVID19. 2020;(April). doi:10.26434/chemrxiv.12115251.v1

17. Yang YY, Wu ZY, Zhang H, et al. LC-MS-based multivariate statistical analysis for the screening of potential thrombin/factor Xa inhibitors from Radix Salvia Miltiorrhiza. Chinese Med (United Kingdom). 2020;15(1):1-13. doi:10.1186/s13020-020-00320-2

18. Heble NK, Mavillapalli RC, Selvaraj R, Jeyabalan S. Molecular docking studies of phytoconstituents identified in Crocus sativus, Curcuma longa, Cassia occidentalis and Moringa oleifera on thymidylate 
synthase - An enzyme target for anti-cancer activity. J Appl Pharm Sci. 2016;6(12):131-135. doi:10.7324/JAPS.2016.601218

19. Jayanandan A, Francis D, Gangadharan AK. Repurposing Simeprevir , Calpain Inhibitor IV and a Cathepsin F Inhibitor Against SARS-CoV-2: A Study Using in Silico Pharmacophore Modeling and Docking Methods. 2020;(May). doi:10.26434/chemrxiv.12317213.v1

20. Khan SU, Htar T. Deciphering the Binding Mechanism of Dexamethasone Against SARS-CoV-2 Main Protease: Computational Molecular Modelling Approach. 2020;(2). doi:10.26434/chemrxiv.12517535.v1

21. Pettersen EF, Goddard TD, Huang CC, et al. UCSF Chimera - A visualization system for exploratory research and analysis. J Comput Chem. 2004;25(13):1605-1612. doi:10.1002/jcc.20084

22. Pavankumar AR, Singh L. Identification of Moringa oleifera protein responsible for the decolorization and pesticide removal from drinking water and industrial effluent - an in silico and in situ evaluation. J Chem Technol Biotechnol. 2015;90(8):1521-1526. doi:10.1002/jctb.4468

23. Vargas JAR, Lopez AG, Piñol MC, Froeyen M. Molecular docking study on the interaction between 2substituted-4,5-difuryl Imidazoles with different protein target for antileishmanial activity. J Appl Pharm Sci. 2018;8(3):14-22. doi:10.7324/JAPS.2018.8303

24. Alagumuthu M, Arumugam S. Molecular explorations of substituted 2 -( 4 - phenylquinolin - 2 - yl ) phenols as phosphoinositide 3 - kinase inhibitors and anticancer agents. Cancer Chemother Pharmacol. 2017. doi:10.1007/s00280-016-3227-z

25. Zhang Y, Mao X, Su J, et al. A network pharmacology-based strategy deciphers the underlying molecular mechanisms of Qixuehe Capsule in the treatment of menstrual disorders. Chinese Med (United Kingdom). 2017;12(1):1-11. doi:10.1186/s13020-017-0145-x

26. Kothandan S, Sasikala RP, Meena KS. Structure based Pharmacophore modeling, Virtual screening and Molecular Docking of Potential Phytochemicals against HSP70. J Appl Pharm Sci. 2017;7(2):137-141. doi:10.7324/JAPS.2017.70218

27. Zhang $Y$, Zhao Z, Kong $P$, et al. A comparative pharmacogenomic analysis of three classic TCM prescriptions for coronary heart disease based on molecular network modeling. Acta Pharmacol Sin. 2020;(July 2019). doi:10.1038/s41401-019-0352-3

28. Liu H, Zhou Q, Wei W, et al. The potential drug for treatment in pancreatic adenocarcinoma: A bioinformatical study based on distinct drug databases. Chinese Med (United Kingdom). 2020;15(1):1-13. doi:10.1186/s13020-020-00309-x

29. Chu $X, Y u T$, Huang $X$, et al. Tomatidine suppresses inflammation in primary articular chondro- cytes and attenuates cartilage degradation in osteoarthritic rats. Aging (Albany NY). 2020;12:1-14.

30. Zuo AR, Dong HH, Yu YY, et al. The antityrosinase and antioxidant activities of flavonoids dominated by the number and location of phenolic hydroxyl groups. Chinese Med (United Kingdom). 2018;13(1):1-12. doi:10.1186/s13020-018-0206-9

31. Seo JY, Kim SK, Nguyen PH, et al. Chemical constituents from a Gynostemma laxum and their antioxidant and neuroprotective activities. Chinese Med (United Kingdom). 2017;12(1):1-13. 
doi:10.1186/s13020-017-0136-y

32. Sathishkumar R, Tharani R. In silico determination of efficiency of plant secondary metabolites to eradicate trachoma - A blinding keratoconjuctivitis disease. J Appl Pharm Sci. 2017;7(9):116-121. doi:10.7324/JAPS.2017.70916

33. Vijayasri S, Hopper W. Towards the identification of novel phytochemical leads as macrodomain inhibitors of Chikungunya virus using molecular docking approach. J Appl Pharm Sci. 2017;7(4):7482. doi:10.7324/JAPS.2017.70410

34. Negi A, Singh P, Taneja N, Mani S. Molecular-docking study of anti-stress natural compounds against GABAa receptor portends the novel approach to stress treatment. J Appl Pharm Sci. 2018;8(12):3843. doi:10.7324/JAPS.2018.81205

35. Ji X, Li Z. Medicinal chemistry strategies toward host targeting antiviral agents. Med Res Rev. 2020; (November 2019):1-39. doi:10.1002/med.21664

36. Stebbing J, Phelan A, Griffin I, et al. COVID-19: combining antiviral and anti-inflammatory treatments. Lancet Infect Dis. 2020;20(4):400-402. doi:10.1016/S1473-3099(20)30132-8

37. Yi F, Tan X lei, Yan X, Liu H bo. In silico profiling for secondary metabolites from Lepidium meyenii (maca) by the pharmacophore and ligand-shape-based joint approach. Chinese Med (United Kingdom). 2016;11(1):1-17. doi:10.1186/s13020-016-0112-y

38. Shyu MH, Kao TC, Yen GC. Oleanolic acid and ursolic acid induce apoptosis in HuH7 human hepatocellular carcinoma cells through a mitochondrial-dependent pathway and downregulation of XIAP. J Agric Food Chem. 2010;58(10):6110-6118. doi:10.1021/jf100574j

\section{Figures}




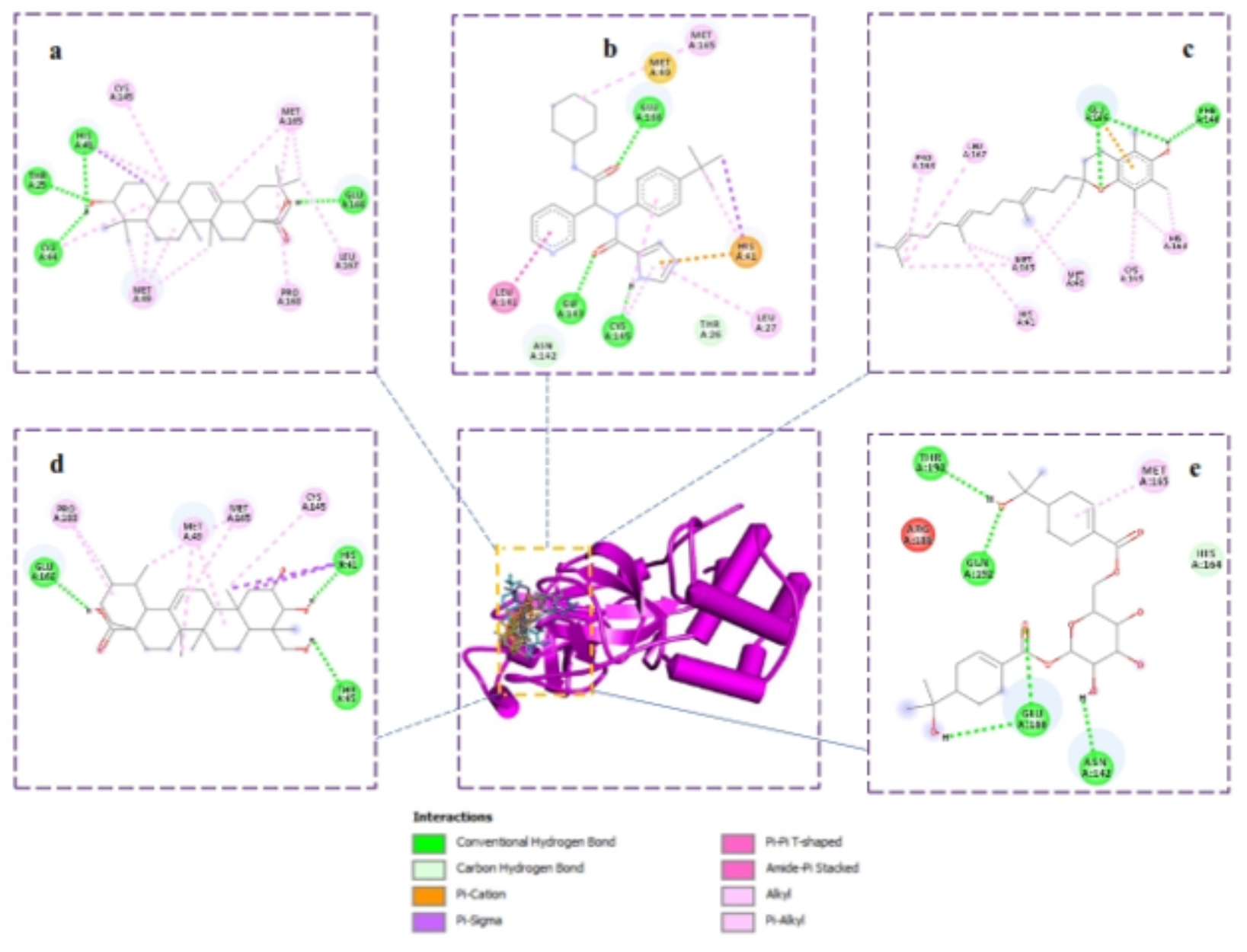

Figure 1

Comparison of 3D structure of four screened compounds docked with the 6 W63 (a)oleanolic acid (b) ligand X77 (c)a-tocotrienol (d)asiatic acid (e)cuniloside 


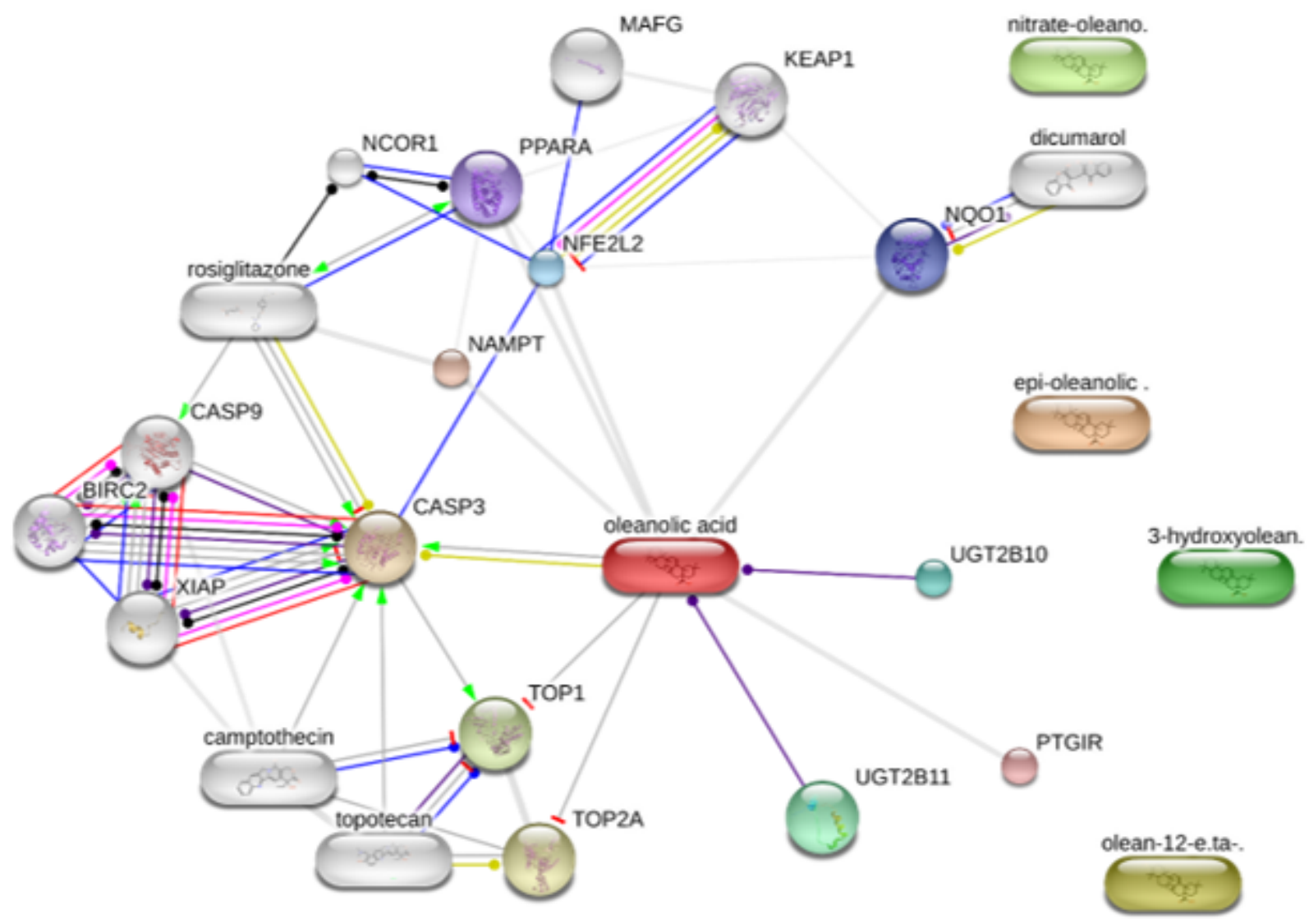

Figure 2

Interaction network of oleanolic acid-target based on STITCH database 